\title{
DIFFUSIVE N-WAVES AND METASTABILITY IN THE BURGERS EQUATION*
}

\author{
YONG JUNG $\mathrm{KIM}^{\dagger}$ AND ATHANASIOS E. TZAVARAS $\ddagger$
}

\begin{abstract}
We study the effect of viscosity on the large time behavior of the viscous Burgers equation by using a transformed version of Burgers (in self-similar variables) that captures efficiently the mechanism of transition to the asymptotic states and allows us to estimate the time of evolution from an $\mathrm{N}$-wave to the final stage of a diffusion wave. Then we construct certain special solutions of diffusive $\mathrm{N}$-waves with unequal masses. Finally, using a set of similarity variables and a variant of the Cole-Hopf transformation, we obtain an integrated Fokker-Planck equation. The latter is solvable and provides an explicit solution of the viscous Burgers equation in a series of Hermite polynomials. This format captures the long-time-small-viscosity interplay, as the diffusion wave and the diffusive $\mathrm{N}$-waves correspond, respectively, to the first two terms in the Hermite polynomial expansion.
\end{abstract}

Key words. diffusion waves, diffusive $\mathrm{N}$-waves, convection-diffusion, metastability

AMS subject classifications. $76 \mathrm{~N} 17,35 \mathrm{~L} 65,76 \mathrm{Rxx}$

PII. S0036141000380516

1. Introduction. The Cauchy problem for the viscous Burgers equation

$$
\begin{array}{r}
u_{t}+u u_{x}=\mu u_{x x}, \quad x \in \mathbb{R}, \mu, t>0, \\
u(x, 0)=u_{0}(x), \quad x \in \mathbb{R},
\end{array}
$$

has, since the pioneering work of Hopf [7], served as a paradigm for the development of the theory of shock waves (see [4] and references therein).

In the limit as the viscosity $\mu \rightarrow 0$, the solution $u^{\mu}$ of (1.1) converges to the entropy weak solution $u$ of the inviscid Burgers

$$
\begin{array}{r}
u_{t}+u u_{x}=0, \quad x \in \mathbb{R}, t>0, \\
u(x, 0)=u_{0}(x), \quad x \in \mathbb{R},
\end{array}
$$

satisfying the Oleinik condition $[16]$

$$
u(x+, t) \leq u(x-, t) .
$$

The asymptotic behavior of (1.2) is an $\mathrm{N}$-wave, whose positive and negative masses are determined by the positive and negative invariants

$$
\begin{gathered}
p_{0}=-\inf _{x} \int_{-\infty}^{x} u_{0} d y, \quad q_{0}=\sup _{x} \int_{x}^{\infty} u_{0} d y, \\
\text { where } \quad-p_{0}+q_{0}=M_{0}=\int_{\mathbb{R}} u_{0}(x) d x,
\end{gathered}
$$

*Received by the editors November 6, 2000; accepted for publication May 3, 2001; published electronically October 4, 2001. This research was partially supported by the National Science Foundation.

http://www.siam.org/journals/sima/33-3/38051.html

${ }^{\dagger}$ Institute for Mathematics and Its Applications, University of Minnesota, Minneapolis, MN 554550436 (yjkim@ima.umn.edu).

$\ddagger$ Department of Mathematics, University of Wisconsin-Madison, Madison, WI 53706 (tzavaras@ math.wisc.edu). 
of the initial data $[7,12]$. On the other hand, the large time behavior of $u^{\mu}$ for fixed $\mu$ is characterized by the well-known diffusion wave of mass $M_{0}$ (see [7]). Therefore, the reversal of order in the successive limit passages $t \rightarrow \infty, \mu \rightarrow 0$ leads to different results; in other words, the long-time response of the viscous Burgers equation exhibits sensitive dependence on the viscosity.

The objective of the present article is to provide a quantitative understanding of the long-time-small-viscosity interplay for the Burgers equation. To place the problem in context, the reader is referred to the numerical runs of section 4 for the evolution of solutions to (1.1), when the viscosity $\mu \ll 1$. These indicate that at an initial stage $u^{\mu}$ evolves from the initial state $u_{0}$ into a saw-tooth profile; at a second stage, the waves interact eventually producing an approximate $\mathrm{N}$-wave; this last structure persists for a very long time, but eventually the smallest of the positive and negative masses is consumed, and thereafter $u^{\mu}$ looks like the final asymptotic state of a diffusion wave.

The Burgers equation is invariant under the group of scaling transformations $x \rightarrow c x, t \rightarrow c^{2} t, u \rightarrow u / c$, and under time and space translations, $t \rightarrow t+a$ and $x \rightarrow x+b$. This property suggests a transformation to similarity variables,

$$
s=\ln (t), \quad \xi=x / \sqrt{t}, \quad w(\xi, s)=\sqrt{t} u(x, t),
$$

which puts (1.1) in the form

$$
w_{s}+\left(\frac{1}{2} w^{2}-\frac{1}{2} \xi w\right)_{\xi}=\mu w_{\xi \xi}, \quad \xi, s \in \mathbb{R}, \quad \mu>0 .
$$

The diffusion waves (see (2.6)) are the steady states of (1.5) and determine its large time behavior (see [7] and sections 2 and 3 ). In the limit $\mu \rightarrow 0$, solutions of (1.5) satisfy the (self-similar variant of the) inviscid Burgers equation

$$
w_{s}+\left(\frac{1}{2} w^{2}-\frac{1}{2} \xi w\right)_{\xi}=0,
$$

subject to the Oleinik entropy condition

$$
w(\xi+, s) \leq w(\xi-, s) .
$$

The admissible steady states of (1.6)-(1.7) are the two parameter family

$$
\mathcal{N}_{p, q}(\xi)= \begin{cases}\xi, & -\sqrt{2 p}<\xi<\sqrt{2 q} \\ 0, & \text { otherwise }\end{cases}
$$

with $p, q$ positive constants. They are precisely the self-similar form of the $\mathrm{N}$-waves, and $p, q$ measure, respectively, the negative and positive mass of the $\mathrm{N}$-wave.

The similarity forms (1.5) and (1.6)-(1.7) provide a convenient formulation for performing long-time numerical runs as well as a framework for a qualitative explanation of the various regimes of the problem. In the first stage the solution evolves from $u_{0}$ to a saw-tooth profile via the usual compression-attenuation mechanism of hyperbolic equations. In the next stage the waves interact and produce an approximate $\mathrm{N}$-wave. In both of these stages the effect of diffusion lies only in selecting admissible discontinuities, and the evolution is essentially governed by the convection equation (1.6)-(1.7). The $\mathrm{N}$-waves are steady states for (1.6), but, due to the presence of small diffusion, they are only approximate solutions for (1.5). This discrepancy drives 
the evolution in the last stage from an approximate $\mathrm{N}$-wave to the steady state of (1.5), a diffusion wave. This stage is a very slow transition and a manifestation of metastability driven by the small diffusion.

A number of techniques have been developed for treating asymptotic behavior problems for nonlinear convection (see, e.g., $[2,3,13]$ ) or convection-diffusion equations (see, e.g., $[5,6,14]$ ). Here we exploit the similarity structure based on the invariances of the Burgers equation. This perspective is initiated in Hopf [7] and is developed in Tartar [20], Liu-Pierre [13] (for convection equations), and EscobedoVazques-Zuazua [5, 6] (for various multidimensional convection-diffusion equations with power laws). The aim is to pursue quantitative explanations of the various regimes; the simplicity of the equation allows us to obtain a complete picture, including the interesting regime of small-viscosity long-time interaction.

We begin, in sections $2-3$, with a review of some of the standard hyperbolic theory of $L^{1}$-contraction and Oleinik inequality for the self-similar Burgers (1.5). We show how to use a special Lyapunov function to establish the asymptotic in time profile (for $\mu$ fixed) of a diffusion wave (see Theorem 3.3). The results in sections $2-3$ can be established with different methods, but we draw attention to the fact that the study of (1.5) provides optimal convergence results with little effort. They also set the stage for section 4 , where we provide explanations and predictions for the numerical runs based on heuristic arguments and theoretical results.

In the last two sections we turn our attention to the issue of metastability. In section 5, we generalize a construction of Whitham [21] and provide a special solution corresponding to a diffusive $\mathrm{N}$-wave with unequal positive and negative masses. This solution reads

$$
\begin{aligned}
u_{p, q}(x, t+1) & =\sqrt{\frac{\mu}{t+1}} v_{p, q}\left(\frac{x}{\sqrt{4 \mu(t+1)}}, t\right), \\
& \text { where } \quad v_{p, q}(\xi, t)=\frac{\frac{B}{\sqrt{\pi}} e^{-\xi^{2}}+2 A \frac{1}{\sqrt{t+1}} \xi e^{-\xi^{2}}}{1-\frac{B}{\sqrt{\pi}} \int_{-\infty}^{\xi} e^{-\zeta^{2}} d \zeta+A \frac{1}{\sqrt{t+1}} e^{-\xi^{2}}},
\end{aligned}
$$

where $B=1-e^{(p-q) / 2 \mu}$ and $A=e^{p / 2 \mu}+O(B)$ are constants determined via the positive and negative masses $p, q$ of the initial data. The solution $u_{p, q}$ converges to an $\mathrm{N}$-wave as $\mu \rightarrow 0$, which suggests the terminology diffusive $\mathrm{N}$-wave for $u_{p, q}$. It captures, in an explicit manner, the slow transition from an approximate $\mathrm{N}$-wave to the final stage of a diffusion wave.

Motivated by the format of (1.8), we use in section 6 appropriate similarity variables and a variant of the Cole-Hopf transformation to transform the Burgers equation into an integrated version of a Fokker-Planck equation. Unlike the Laplace operator on the real line, the present operator (6.7) has a discrete spectrum. The process yields an explicit formula for the solution of the Burgers equations in terms of Hermite polynomials:

$$
\begin{aligned}
& u(x, t)=\sqrt{\frac{\mu}{t+1}} v\left(\frac{x}{\sqrt{4 \mu(t+1)}}, t\right), \\
& \text { where } v(\xi, t)=-\frac{\partial_{\xi} \psi_{M}^{\infty}+\sum_{n=0}^{\infty} a_{n}(t+1)^{-\frac{n+1}{2}} \partial_{\xi}\left(H_{n}(\xi) e^{-\xi^{2}}\right)}{\psi_{M}^{\infty}+\sum_{n=0}^{\infty} a_{n}(t+1)^{-\frac{n+1}{2}} H_{n}(\xi) e^{-\xi^{2}}},
\end{aligned}
$$

where $\psi_{M}^{\infty}$ is the potential of a diffusion wave of mass $M$ (see (6.16)), $H_{n}$ are the Hermite polynomials, and the Fourier-Hermite coefficients $a_{n}$ are computed explicitly 
from the data (see (6.18)). The asymptotic profile of the diffusion wave corresponds to keeping only the first term in the expansion, $-\frac{\partial_{\xi} \psi_{M}^{\infty}}{\psi_{M}^{\infty}}$, while the diffusive N-wave (1.8) corresponds to keeping the first two terms in the expansion and describes the first order correction of the general solution beyond the diffusion wave.

2. Self-similar Burgers - preliminaries. Some of the basic properties of the viscous Burgers equations are directly linked to the self-similar Burgers equation

$$
w_{s}+\left(\frac{1}{2} w^{2}-\frac{1}{2} \xi w\right)_{\xi}=\mu w_{\xi \xi}
$$

This problem is obtained from (1.1) in two different ways. If the similarity transformation (1.4) is used, then one obtains (2.1a) set in the interval $(\xi, s) \in \mathbb{R} \times \mathbb{R}$, and the initial data are projected back to $-\infty$. Alternatively, and due to the invariance $t \rightarrow t+1$, one may use the transformation

$$
s=\ln (t+1), \quad \xi=x / \sqrt{t+1}, \quad w(\xi, s)=\sqrt{t+1} u(x, t)
$$

and obtain the initial value problem consisting of $(2.1 \mathrm{a})$ set on $(\xi, s) \in \mathbb{R} \times \mathbb{R}^{+}$subject to data

$$
w(\xi, 0)=w_{0}(\xi)
$$

In either case the total mass remains invariant under the transformation, and the initial mass is preserved:

$$
\int_{\mathbb{R}} w(\xi, s) d \xi=M_{0}<\infty
$$

Let $w^{\mu}$ be the solution of (2.1). In the limit $\mu \rightarrow 0, w^{\mu} \rightarrow w$ a.e., where $w$ is a weak solution of the initial value problem

$$
\begin{array}{r}
w_{s}+\left(\frac{1}{2} w^{2}-\frac{1}{2} \xi w\right)_{\xi}=0, \\
w(\xi, 0)=w_{0}(\xi),
\end{array}
$$

that satisfies the Oleinik (entropy) condition

$$
w(\xi+, s) \leq w(\xi-, s) .
$$

2.1. Steady states. The admissible steady states of (2.2) are given by the two parameter family

$$
\mathcal{N}_{p, q}(\xi)= \begin{cases}\xi, & -\sqrt{2 p}<\xi<\sqrt{2 q} \\ 0, & \text { otherwise }\end{cases}
$$

parametrized by the positive constants $p$ and $q$ measuring, respectively, the mass of the negative and positive parts of the steady state. These solutions are precisely the $\mathrm{N}$-waves, when viewed on the self-similar coordinates, and are denoted by $\mathcal{N}_{p, q}$. If the total mass $M$ is prescribed, there is a one parameter family $\mathcal{N}_{p, p+M}$ corresponding to the mass $M$. 
The equilibria of (2.1a) (for $\mu>0$ ) satisfy the equation

$$
\left(\frac{1}{2} \mathcal{G}^{2}-\frac{1}{2} \xi \mathcal{G}\right)^{\prime}=\mu \mathcal{G}^{\prime \prime}
$$

and are computed by the formula

$$
\mathcal{G}_{M}(\xi)=\frac{\sqrt{\mu}\left(1-e^{-M / 2 \mu}\right) e^{-\xi^{2} / 4 \mu}}{1-\left(1-e^{-M / 2 \mu}\right) \frac{1}{\sqrt{\pi}} \int_{-\infty}^{\xi / \sqrt{4 \mu}} e^{-\zeta^{2}} d \zeta}, \quad M \in \mathbb{R} .
$$

The denominator in (2.6) does not vanish, and the total mass of $\mathcal{G}_{M}$ is computed by

$$
\int_{-\infty}^{\infty} \mathcal{G}_{M} d \xi=-2 \mu \int_{-\infty}^{\infty} \partial_{\xi} \ln \left(1-\left(1-e^{-M / 2 \mu}\right) \frac{1}{\sqrt{\pi}} \int_{-\infty}^{\xi / \sqrt{4 \mu}} e^{-\zeta^{2}} d \zeta\right) d \xi=M
$$

The steady states of the viscous problem are thus determined by the total mass $M$ and correspond to the well-known diffusion waves in self-similar coordinates. We summarize some of their properties below.

Lemma 2.1. Let $\mathcal{G}_{M}$ be a diffusion wave given by (2.6). Then

(i) $\mathcal{G}_{0}(\xi)=0$ and $\int_{-\infty}^{\infty} \mathcal{G}_{M} d \xi=M$;

(ii) $\mathcal{G}_{M}(\xi)>\mathcal{G}_{M^{\prime}}(\xi)$ for $M>M^{\prime}$;

(iii) for any bounded function $f(\xi)$ with a compact support, there exists $M>0$ such that $\mathcal{G}_{-M}(\xi) \leq f(\xi) \leq \mathcal{G}_{M}(\xi)$;

(iv) $\mathcal{G}_{M} \rightarrow \mathcal{N}_{0, M}$ as $\mu \rightarrow 0$ for $M>0$ and $\mathcal{G}_{M} \rightarrow \mathcal{N}_{|M|, 0}$ as $\mu \rightarrow 0$ for $M<0$.

2.2. Oleinik inequality. We present a quick derivation of some well-known properties viewed from the perspective of (2.1a). Recall that (2.1a) arises from the transformation (1.4) and is set on $\mathbb{R} \times \mathbb{R}$. The derived estimates are independent of $\mu$ and $s$. We begin with the analogue of the Oleinik estimate [16].

LEMma 2.2. The solution $w(\xi, s)$ of (2.1a) satisfies

$$
w_{\xi}(\xi, s) \leq 1, \quad s, \xi \in \mathbb{R} .
$$

Proof. The quantity $z=w_{\xi}$ satisfies

$$
z_{s}+\left(w-\frac{1}{2} \xi\right) z_{\xi}+z(z-1)=\mu z_{\xi \xi} .
$$

If $z$ has an interior maximum, then the value at the maximum is between 0 and 1 . Since

$$
w_{\xi}(\xi, \ln t)=t u_{x}(\sqrt{t} \xi, t),
$$

for smooth data $u_{0}$ we have $\lim _{s \rightarrow-\infty} w_{\xi}(\xi, s)=0$, and (2.8) follows. If the data are not smooth, they may be approximated by smooth data in a standard way, and we conclude (2.8) by a density argument.

Consider the functions

$$
\begin{aligned}
W(\xi, s)= & W_{-}(\xi, s)=\int_{-\infty}^{\xi} w(\zeta, s) d \zeta \\
W_{+}(\xi, s) & =\int_{\xi}^{\infty} w(\zeta, s) d \zeta=M_{0}-W(\xi, s)
\end{aligned}
$$


and the quantities

$$
\begin{aligned}
& p(s)=-\inf _{\xi} W(\xi, s), \\
& q(s)=\sup _{\xi} W_{+}(\xi, s)=M_{0}+p(s) .
\end{aligned}
$$

$p(s)$ and $q(s)$ are time-invariants for solutions of the inviscid problem [12], but for the viscous problem they do not remain constant anymore. $W$ satisfies a viscous Hamilton-Jacobi equation

$$
W_{s}+\frac{1}{2}\left(W_{\xi}-\xi\right) W_{\xi}=\mu W_{\xi \xi} .
$$

As a simple implication of the maximum principle and Lemma 2.2, we have the following lemma.

Lemma 2.3. Let $w(\xi, s ; \mu)$ be the solution of $(2.1 \mathrm{a})$, let $W$ be its integral given by (2.11), and let $A=-\inf W(\xi, 0)$ and $B=\sup W(\xi, 0) \geq 0$. Then $W$ and $w$ are uniformly bounded by

$$
\begin{gathered}
-A \leq W(\xi, s) \leq B, \\
|w(\xi, s)| \leq \sqrt{2(A+B)} .
\end{gathered}
$$

Proof. The estimate (2.14) follows from the maximum principle. To show (2.15), suppose that $w\left(\xi_{1}, s\right)>\sqrt{2(A+B)}$ for some $s, \xi_{1}$. Let $\xi_{0}<\xi_{1}$ be such that $w\left(\xi_{0}\right)=0$ and $w(\xi, s)>0$ on $\left(\xi_{0}, \xi_{1}\right)$. (If $w>0$ on $\left(-\infty, \xi_{1}\right)$, we take $\xi_{0}=-\infty$.) Since $w_{\xi} \leq 1$, we have $\int_{\xi_{0}}^{\xi_{1}} w(\xi, s) d \xi>A+B$ and

$$
W\left(\xi_{1}, s\right)=W\left(\xi_{0}, s\right)+\int_{\xi_{0}}^{\xi_{1}} w(\xi, s) d \xi>B
$$

thus violating (2.15). If it is assumed that $w\left(\xi_{1}, s\right)<-\sqrt{2(A+B)}$, then similar arguments lead to a contradiction.

2.3. $L^{1}$-contraction theory. From now on, consider the initial value problem (2.1) consisting of (2.1a) and (2.1b) and set $(\xi, s) \in \mathbb{R} \times \mathbb{R}^{+}$. For data $w_{0} \in L^{\infty} \cap L^{1}$ this problem has a unique smooth solution. Let $w_{1}, w_{2}$ be two solutions; their difference $v=w_{1}-w_{2}$ satisfies the linear parabolic equation

$$
\begin{array}{r}
v_{s}+\frac{1}{2}\left(\left(w_{1}+w_{2}-\xi\right) v\right)_{\xi}=\mu v_{\xi \xi}, \\
v(\xi, 0)=w_{1}(\xi, 0)-w_{2}(\xi, 0) .
\end{array}
$$

The integral

$$
V(\xi, s)=\int_{-\infty}^{\xi} v(\zeta, s) d \zeta=\int_{-\infty}^{\xi} w_{1}(\zeta, s)-w_{2}(\zeta, s) d \zeta
$$

satisfies

$$
V_{s}+\frac{1}{2}\left(w_{1}+w_{2}-\xi\right) V_{\xi}-\mu V_{\xi \xi}=0
$$


Let $v^{+}=\max \{v, 0\}=(v+|v|) / 2$.

THEOREM 2.4. Let $w_{1}, w_{2}$ be classical solutions of $(2.1)$ with initial data $w_{1}(\xi, 0)$ and $w_{2}(\xi, 0)$. Then

$$
\begin{gathered}
\int_{-\infty}^{\infty}\left|w_{1}(\xi, s)-w_{2}(\xi, s)\right| d \xi \leq \int_{-\infty}^{\infty}\left|w_{1}(\xi, 0)-w_{2}(\xi, 0)\right| d \xi \\
\int_{-\infty}^{\infty}\left(w_{1}(\xi, s)-w_{2}(\xi, s)\right)^{ \pm} d \xi \leq \int_{-\infty}^{\infty}\left(w_{1}(\xi, 0)-w_{2}(\xi, 0)\right)^{ \pm} d \xi .
\end{gathered}
$$

Furthermore, the quantity

$$
\int_{-\infty}^{\infty}\left(w_{1}(\xi, s)-w_{2}(\xi, s)\right)^{ \pm} d \xi
$$

decreases strictly in $s$, unless either $w_{1}(\xi, s) \leq w_{2}(\xi, s)$ or $w_{2}(\xi, s) \leq w_{1}(\xi, s)$ for all $\xi \in \mathbb{R}$.

Proof. The first part is a direct consequence of the standard contraction theory for convection-diffusion equations; see $[8,18]$. We present a proof of the second part, which is based on a detailed analysis of a linear equation

$$
v_{s}+(a(\xi, s) v)_{\xi}=\mu v_{\xi \xi},
$$

with $a(\xi, s)$ continuously differentiable and $\left|a_{\xi}\right| \leq M$.

Step 1. Let $v^{+}=\max \{v, 0\}=\frac{v+|v|}{2}$. Then $\partial_{\xi} v^{+}=v_{\xi} \chi_{\{v>0\}}, \partial_{s} v^{+}=v_{s} \chi_{\{v>0\}}$, and $v^{+} \in W^{1, \infty}\left(\mathbb{R} \times \mathbb{R}^{+}\right)$. Also set

$$
V^{+}(\xi, s)=\int_{-\infty}^{\xi} v^{+}(\zeta, s) d \zeta=\int_{-\infty}^{\xi}\left(w_{1}-w_{2}\right)^{+}(\zeta, s) d \zeta
$$

which (due to the integrability of $v$ and $v_{s}$ ) enjoys the regularity $W^{2, \infty}$ in $\xi$ and $W^{1, \infty}$ in $s$. We show in this step that $v^{+}$and $V^{+}$satisfy

$$
\begin{gathered}
\left(v^{+}\right)_{s}+\left(a(\xi, s) v^{+}\right)_{\xi} \leq \mu\left(v^{+}\right)_{\xi \xi}, \\
\left(V^{+}\right)_{s}+a(\xi, s)\left(V^{+}\right)_{\xi} \leq \mu\left(V^{+}\right)_{\xi \xi}
\end{gathered}
$$

in the sense of distributions.

To see that, consider the functions

$$
\psi_{n}(v)=\left\{\begin{array}{rr}
0, & v \leq 0, \\
n v, & 0 \leq v \leq 1 / n, \\
1, & v \geq 1 / n,
\end{array} \quad \Psi_{n}(v)=\int_{-\infty}^{v} \psi_{n}(\tau) d \tau=\left\{\begin{array}{rr}
0, & v \leq 0 \\
\frac{n v^{2}}{2}, & 0 \leq v \leq 1 / n \\
v-\frac{1}{2 n}, & v \geq 1 / n
\end{array}\right.\right.
$$

Then $\Psi_{n}(v)$ satisfies

$$
\begin{aligned}
\left(\Psi_{n}(v)\right)_{s}+\left(a \Psi_{n}(v)\right)_{\xi}+a_{\xi}\left(v \psi_{n}(v)-\Psi_{n}(v)\right) & =\mu\left(\Psi_{n}(v)\right)_{\xi \xi}-\mu \psi_{n}^{\prime}(v) v_{\xi}^{2} \\
& \leq \mu\left(\Psi_{n}(v)\right)_{\xi \xi} .
\end{aligned}
$$


Using the properties

$$
\begin{array}{r}
\Psi_{n}(v) \rightarrow v^{+}, \quad 0 \leq \Psi_{n}(v) \leq v^{+}, \\
v \psi_{n}(v)-\Psi_{n}(v) \rightarrow 0, \quad\left|v \psi_{n}(v)-\Psi_{n}(v)\right| \leq \frac{1}{2} v^{+},
\end{array}
$$

we pass to the limit $n \rightarrow \infty$ in (2.25) and obtain (2.23).

Next we consider the integrated version of (2.25),

$$
\begin{aligned}
\partial_{s} \int_{-\infty}^{\xi} \Psi_{n}(v) d \zeta & +a \Psi_{n}(v)+\int_{-\infty}^{\xi} a_{\xi}\left(v \psi_{n}(v)-\Psi_{n}(v)\right) d \zeta \\
& =\mu \partial_{\xi} \Psi_{n}(v)-\mu \int_{-\infty}^{\xi} \psi_{n}^{\prime}(v) v_{\xi}^{2} d \zeta \leq \mu \partial_{\xi} \Psi_{n}(v),
\end{aligned}
$$

and use the properties (2.25), $\left|a_{\xi}\right| \leq M$, and

$$
\begin{array}{r}
\int_{-\infty}^{\xi} \Psi_{n}(v) d \zeta \rightarrow \int_{-\infty}^{\xi} v^{+} d \zeta, \quad \int_{-\infty}^{\xi} \Psi_{n}(v) d \zeta \leq V^{+} \\
\left|\int_{-\infty}^{\xi} a_{\xi}\left(v \psi_{n}(v)-\Psi_{n}(v)\right) d \zeta\right| \leq \frac{M}{2} \int_{-\infty}^{\xi} v^{+} d \zeta
\end{array}
$$

to pass to the limit $n \rightarrow \infty$ and derive (2.24).

Step 2. From (2.23) we derive the inequality

$$
\frac{d}{d s} \int \varphi(\xi) v^{+}(\xi, s) d \xi-\int\left(a v^{+}-\mu\left(v^{+}\right)_{\xi}\right)(\xi, s) \varphi_{\xi}(\xi) d \xi \leq 0
$$

for any positive test function $\varphi \in C_{c}^{\infty}(\mathbb{R})$. In turn, this yields that $\int v^{+}(\xi, s) d \xi$ is decreasing in $s$. Since $\int v(\xi, s) d \xi$ is a conserved quantity, this implies (2.18) and (2.19).

We may obtain a more detailed variant of (2.19) as follows. Fix $s>0$ and consider a decomposition of the open set $\{\xi: v(\xi, s)>0\}=\cup_{k}\left(\alpha_{k}, \beta_{k}\right)$ into countably many subintervals such that $v(\cdot, s)>0$, and $C^{1}$ on $\left(\alpha_{k}, \beta_{k}\right), v_{\xi}\left(\alpha_{k}, s\right) \geq 0$, and $v_{\xi}\left(\beta_{k}, s\right) \leq 0$. Consider a test function $\varphi_{n}$ such that

$$
\varphi_{n}(\xi)=\left\{\begin{aligned}
0, & \xi \leq \alpha_{k} \text { or } \xi \geq \beta_{k}, \\
1, & \alpha_{k}+\frac{1}{n} \leq \xi \leq \beta_{k}-\frac{1}{n} \\
\text { linear, } & \alpha_{k}<\xi<\alpha_{k}+\frac{1}{n} \text { or } \beta_{k}-\frac{1}{n}<\xi<\beta_{k} .
\end{aligned}\right.
$$

We apply (2.29) for this test function and pass to the limit $n \rightarrow \infty$ to obtain

$$
\frac{d}{d s} \int_{\alpha_{k}}^{\beta_{k}} v^{+}(\xi, s) d \xi \leq \mu\left(v_{\xi}\left(\beta_{k}, s\right)-v_{\xi}\left(\alpha_{k}, s\right)\right) \leq 0,
$$

$k=1,2, \ldots$; that is, the area under any component of $v^{+}$is decreasing in size.

Step 3. The second part of the theorem, which is a stronger version of the first part, is obtained from the strong maximum principle. Consider $V^{+}(\xi, s)$ in $(2.22)$, fix $\bar{s}>0$, and suppose there exists $\xi_{0}<\xi_{1}$ such that $v\left(\xi_{0}, \bar{s}\right)>0$ and $v\left(\xi_{1}, \bar{s}\right)<0$. Consider a restriction of $V^{+}$defined by

$$
Z^{+}(\xi, s)=\int_{-\infty}^{\xi} v^{+}(\zeta, s) \chi_{\left(-\infty, \xi_{1}\right)}(\zeta) d \zeta=\left\{\begin{array}{ll}
V^{+}(\xi, s) & \text { if } \xi<\xi_{1}, \\
V^{+}\left(\xi_{1}, s\right) & \text { if } \xi>\xi_{1},
\end{array} \quad|s-\bar{s}|<\delta\right.
$$


where

$$
\chi_{\left(-\infty, \xi_{1}\right)}= \begin{cases}1, & \xi \in\left(-\infty, \xi_{1}\right), \\ 0, & \xi \notin\left(-\infty, \xi_{1}\right) .\end{cases}
$$

We can take $\delta>0$ so small that $Z^{+}$has the same regularity as $V^{+}$and compute

$$
\begin{aligned}
\partial_{s} Z^{+}+ & a \partial_{\xi} Z^{+}-\mu \partial_{\xi}^{2} Z^{+} \\
& =\left(\partial_{s} V^{+}+a \partial_{\xi} V^{+}-\mu \partial_{\xi}^{2} V^{+}\right) \chi_{\left(-\infty, \xi_{1}\right)}-\mu v^{+}\left(\xi_{1}, s\right) \delta\left(\xi-\xi_{1}\right) \\
& +\partial_{s} V^{+}\left(\xi_{1}, s\right) \chi_{\left(-\infty, \xi_{1}\right)}=I_{1}+I_{2}+I_{3} \leq 0
\end{aligned}
$$

where the last inequality follows from $(2.24)$ and the properties $v^{+}\left(\xi_{1}, s\right)=0$ (hence $I_{2}=0$ ) and (from step 2)

$$
\frac{d}{d s} \int_{-\infty}^{\xi_{1}} v^{+}(\zeta, s) d \zeta=\frac{d}{d s} V^{+}\left(\xi_{1}, s\right) \leq 0
$$

Therefore, $Z^{+}$satisfies the parabolic inequality (2.30) in the interval $0<s-\bar{s}<\delta$.

If $Z^{+}\left(\xi_{1}, s\right)$ is strictly decreasing at $\bar{s}$, then (2.20) follows. On the other hand, if $Z^{+}\left(\xi_{1}, s\right)$ remains constant in the small time interval, then $Z^{+}(\cdot, s)$ has a strictly positive maximum at the interior point $\xi=\xi_{1}$ for a time interval $0<s-\bar{s}<\delta$ which contradicts the strong maximum principle (see, e.g., [11]).

Remark 2.5 (the lap-number). For semilinear parabolic equations there exists a literature concerning the number of zeroes of a solution, sometimes called the lapnumber (see, e.g., Matano [15] and Angenent [1]). Such results hinge on analysis of the linear equation

$$
u_{t}=u_{x x}+q(x, t) u, \quad x \in \mathbb{R}, t>0,
$$

when $q(x, t) \in L^{\infty}$ and for solutions satisfying the bound $|u(x, t)| \leq A e^{B x^{2}}$. It is shown in [1] that the number of zeroes

$$
\mathbb{Z}_{t}=\{x \in \mathbb{R}: u(x, t)=0\}
$$

becomes immediately (at time $t=0+$ ) a discrete set, and thereafter the number of zeroes is decreasing in time. The basis of the last result is the property that if $u(x, t)$ and $u_{x}(x, t)$ vanish simultaneously at $\left(x_{0}, t_{0}\right)$ (i.e., $u$ has a multiple zero at $\left(x_{0}, t_{0}\right)$ ), then, roughly speaking, $u(\cdot, t)$ has more zeroes for $t<t_{0}$ than for $t>t_{0}$ (see Angenent $[1$, Theorem B] for the precise statement).

These results apply to various semilinear parabolic equations that can (through transformations of variables) be put in the form (2.31) [1]. They also apply to quasilinear equations when linear variants of them can be put into this framework. In particular, the viscous Burgers equation shares this property (along solutions that the wave speed $u(x, t)$ and its first derivatives are uniformly bounded). Finally, the same property is transferred to solutions $w(\xi, s)$ of $(2.1)$ through the similarity transformations.

3. Evolution of the viscous problem. Here we study the long-time convergence of solutions to a diffusion wave. 
3.1. Convergence to a diffusion wave. Consider the difference $w(\xi, s)-$ $\mathcal{G}_{M}(\xi)$, and note that the $L^{1}$-contraction implies

$$
\int_{-\infty}^{\infty}\left(w(\xi, s)-\mathcal{G}_{M}(\xi)\right)^{ \pm} d \xi \leq \int_{-\infty}^{\infty}\left(w(\xi, 0)-\mathcal{G}_{M}(\xi)\right)^{ \pm} d \xi
$$

First, we prove a technical lemma, indicating that if the solution stabilizes, then the mass of the solution stabilizes.

LEMma 3.1. Let $w$ be the solution of (2.1) emanating from initial data $w_{0} \in$ $L^{1} \cap L^{\infty}$. If along a time sequence $\left\{s_{k}\right\}$ we have $w\left(\xi, s_{k}\right) \rightarrow \bar{w}(\xi)$ a.e. $\xi$ as $s_{k} \rightarrow \infty$, then

$$
\lim _{s_{k} \rightarrow \infty} \int_{-\infty}^{\infty} w\left(\zeta, s_{k}\right) d \zeta=\int_{-\infty}^{\infty} \lim _{s_{k} \rightarrow \infty} w\left(\zeta, s_{k}\right) d \zeta
$$

Proof. Assume first that the data satisfy

$$
\mathcal{G}_{-M}(\xi) \leq w_{0}(\xi) \leq \mathcal{G}_{M}(\xi) .
$$

Then from the comparison estimate (2.19)

$$
\mathcal{G}_{-M}(\xi) \leq w(\xi, s) \leq \mathcal{G}_{M}(\xi)
$$

and the dominated convergence theorem implies the desired result.

Now let $w_{0} \in L^{1} \cap L^{\infty}$. For $\varepsilon>0$ choose $M>0$ so that

$$
\int_{-\infty}^{\infty}\left(w_{0}(\zeta)-\mathcal{G}_{M}(\zeta)\right)^{+}+\left(w_{0}(\zeta)-\mathcal{G}_{-M}(\zeta)\right)^{-} d \zeta<\varepsilon
$$

Let

$$
w_{M}(\xi, s)= \begin{cases}\mathcal{G}_{M}(\xi) & \text { if } w>\mathcal{G}_{M}, \\ w(\xi, s) & \text { if } \mathcal{G}_{-M}<w<\mathcal{G}_{M}, \\ \mathcal{G}_{-M}(\xi) & \text { if } w<\mathcal{G}_{-M}\end{cases}
$$

and define $\bar{w}_{M}(\xi)$ in a similar fashion using the limit function $\bar{w}(\xi)$ in the place of $w(\xi, s)$. Observe that $w_{M}\left(\zeta, s_{k}\right) \rightarrow \bar{w}_{M}(\zeta)$ and that

$$
\begin{aligned}
& \left|\int_{-\infty}^{\infty}\left(w\left(\zeta, s_{k}\right)-w_{M}\left(\zeta, s_{k}\right)\right) d \zeta\right| \\
& \quad=\left|\int_{w>\mathcal{G}_{M}}\left(w\left(\zeta, s_{k}\right)-\mathcal{G}_{M}(\zeta)\right) d \zeta+\int_{w<\mathcal{G}_{-M}}\left(w\left(\zeta, s_{k}\right)-\mathcal{G}_{-M}(\zeta)\right) d \zeta\right| \leq 2 \varepsilon
\end{aligned}
$$

from (2.19), (3.1), and (3.3). Now $\int_{\mathbb{R}} w_{M}\left(\cdot, s_{k}\right) d \zeta \rightarrow \int_{\mathbb{R}} \bar{w}_{M} d \zeta$, and we conclude by $(3.3)$

$$
\left|\lim _{s_{k} \rightarrow \infty} \int_{-\infty}^{\infty} w\left(\zeta, s_{k}\right) d \zeta-\int_{-\infty}^{\infty} \bar{w}(\zeta) d \zeta\right| \leq 4 \varepsilon,
$$

which gives (3.2).

In the following theorem we show that the solution of (2.1) converges to a diffusion wave preserving the initial total mass. 
TheOREm 3.2 (convergence in time). Let $w$ be the solution to the Cauchy problem (2.1) with $\mu>0$ and $w_{0} \in L^{1} \cap L^{\infty}, \partial_{\xi} w_{0} \in L^{2}$, and total mass $\int w_{0}(\xi) d \xi=M_{0}$. Then

$$
w(\xi, s) \rightarrow \mathcal{G}_{M_{0}}(\xi) \text { as } s \rightarrow \infty,
$$

a.e. and in $L^{1}(\mathbb{R})$, where $\mathcal{G}_{M_{0}}$ is the diffusion wave given by $(2.6)$.

Proof. Consider the quantity $\Phi=e^{-\frac{1}{2 \mu} W}$ motivated by the Cole-Hopf transformation. Then $\Phi$ satisfies

$$
\Phi_{s}-\frac{1}{2} \xi \Phi_{\xi}=\mu \Phi_{\xi \xi}
$$

We differentiate (3.6) with respect to $s$ and multiply by $\Phi_{s}$. After rearranging the terms, we obtain

$$
\partial_{s} \Phi_{s}^{2}-\frac{1}{2} \partial_{\xi}\left(\xi \Phi_{s}^{2}\right)+\frac{1}{2} \Phi_{s}^{2}+2 \mu \Phi_{\xi s}^{2}=\mu \partial_{\xi \xi} \Phi_{s}^{2}
$$

Therefore, the quantity

$$
g(s)=\int_{\mathbb{R}} \Phi_{s}^{2}(\xi, s) d \xi=\int_{\mathbb{R}} \frac{1}{4 \mu^{2}} e^{-\frac{1}{\mu} W} W_{s}^{2} d \xi
$$

satisfies the differential inequality $\frac{d g}{d s}+\frac{1}{2} g \leq 0$. We conclude that

$$
\int_{\mathbb{R}} e^{-\frac{1}{\mu} W} W_{s}^{2} d \xi \leq\left(\int_{\mathbb{R}} e^{-\frac{1}{\mu} W(\xi, 0)} W_{s}^{2}(\xi, 0) d \xi\right) e^{-\frac{s}{2}}
$$

and, from (2.14) and (2.13), that

$$
\int_{\mathbb{R}} W_{s}^{2}(\xi, s) d \xi \leq O(1) e^{-\frac{s}{2}}
$$

where $O(1)$ depends on $\mu$ and the $H^{1}$-norm of the data $w_{0}$. (The last dependence may be relaxed by using the regularizing effect of (2.1), but we will not pursue the details here.)

From $(2.8)$ and $(2.15)$, the function $(w(\xi, s)-\xi)$ is decreasing and for any $[a, b] \subset \mathbb{R}$ we have

$$
T V_{\xi \in[a, b]} w(\cdot, s) \leq \sup _{\xi} w_{0}-\inf _{\xi} w_{0}+2(b-a)
$$

From Helly's theorem and a diagonal argument we can extract a subsequence $s_{n} \rightarrow \infty$ and a function $\bar{w}$ of locally bounded variation so that

$$
w\left(\xi, s_{n}\right) \rightarrow \bar{w}(\xi) \quad \text { as } s_{n} \rightarrow \infty .
$$

Let $\theta(\xi)$ be a $C^{\infty}$-function with compact support. From (2.13) we obtain

$$
\int_{\mathbb{R}} W_{s}(\cdot, s) \theta d \xi+\int_{\mathbb{R}} \frac{1}{2}\left(w^{2}-\xi w\right) \theta d \xi+\int_{\mathbb{R}} \mu w \theta_{\xi} d \xi=0 .
$$

We use (3.7), (3.8), and Lemma 3.1 to pass to the limit along $s_{n}$ and deduce that $\bar{w}$ satisfies

$$
\begin{aligned}
\frac{1}{2} \bar{w}^{2}-\frac{1}{2} \xi \bar{w} & =\mu \bar{w}_{\xi}, \\
\int_{\mathbb{R}} \bar{w} d \xi & =\int_{\mathbb{R}} w_{0} d \xi=M_{0} .
\end{aligned}
$$

Therefore, $\bar{w}=\mathcal{G}_{M_{0}}$, and, as the limit is uniquely determined by (3.9), we conclude that the family $w(\xi, s) \rightarrow \mathcal{G}_{M_{0}}(\xi)$ as $s \rightarrow \infty$. 
3.2. Convergence to a diffusion wave via the invariance principle. Next we provide a proof of the time-convergence to a diffusion wave from the viewpoint of the LaSalle invariance principle, in the spirit of $[3,6]$. Recall that the solution operator of the viscous Burgers defines a contraction semigroup in $L^{1}(\mathbb{R})$ defined by $T(s) w_{0}=w(\cdot, s)$, where $w_{0} \in L^{1}(\mathbb{R}) \cap L^{\infty}(\mathbb{R})$ is the initial datum.

TheOREM 3.3 (convergence in time). Let $w$ be the solution to the Cauchy problem (2.1) with $\mu>0$ and $w_{0} \in L^{1} \cap L^{\infty}$ with total mass $\int w_{0}(\xi) d \xi=M_{0}$. Then

$$
w(\xi, s) \rightarrow \mathcal{G}_{M_{0}}(\xi) \quad \text { as } s \rightarrow \infty,
$$

pointwise and in $L^{p}(\mathbb{R}), 1 \leq p<\infty$, where $\mathcal{G}_{M_{0}}$ is the diffusion wave given by (2.6).

Proof. Consider the $\omega$-limit set

$$
\omega\left(w_{0}\right)=\left\{\psi \in L^{1}(\mathbb{R}): \psi=\lim _{k \rightarrow \infty} T\left(s_{k}\right) w_{0} \text { for a subsequence } s_{k}\right\} .
$$

From (3.8) we have a convergent subsequence $T\left(s_{k}\right) w_{0}=w\left(\cdot, s_{k}\right)$ and thus $\omega\left(w_{0}\right)$ is not empty. Moreover, $T$ is positively invariant; that is, if $\psi \in \omega\left(w_{0}\right)$, then $T(s) \psi \in \omega\left(w_{0}\right)$. This follows from the semigroup property and the observation

$$
T(s) \psi=\lim _{k \rightarrow \infty} T(s) T\left(s_{k}\right) w_{0}=\lim _{k \rightarrow \infty} T\left(s+s_{k}\right) w_{0} .
$$

Consider the distance functional $V: L^{1}(\mathbb{R}) \rightarrow \mathbb{R}$ defined by

$$
V(\chi)=\left\|\chi-\mathcal{G}_{M_{0}}\right\|_{L^{1}}
$$

which is continuous in $L^{1}(\mathbb{R})$. The diffusion wave $\mathcal{G}_{M_{0}}$ is a special solution of (2.1). Due to the contraction theory (2.18), $V$ satisfies

$$
V\left(T(s) w_{0}\right)=\left\|w(\cdot, s)-\mathcal{G}_{M_{0}}\right\|_{L^{1}} \leq\left\|w_{0}-\mathcal{G}_{M_{0}}\right\|_{L^{1}}=V\left(w_{0}\right)
$$

for any $s>0, w_{0} \in L^{1}(\mathbb{R})$. Since $V\left(T(s) w_{0}\right)$ is decreasing in time, it converges $V\left(T(s) w_{0}\right) \rightarrow c$ to a limit $c \geq 0$. From the continuity of $V$, we obtain

$$
V(T(s) \psi)=V\left(\lim _{k \rightarrow \infty} T\left(s+s_{k}\right) w_{0}\right)=\lim _{k \rightarrow \infty} V\left(T\left(s+s_{k}\right) w_{0}\right)=c
$$

for any $\psi \in \omega\left(w_{0}\right)$ and $s \geq 0$.

We shall assume there is $\psi \in \omega\left(w_{0}\right)$ such that $\psi \neq \mathcal{G}_{M_{0}}$ and establish a contradiction. For such a $\psi$ we have $V(\psi)=c>0, T(s) \psi \in \omega\left(w_{0}\right)$, and $V(T(s) \psi)=c$ from (3.15). Since

$$
\int \psi(\xi)-\mathcal{G}_{M_{0}}(\xi) d \xi=0,
$$

there exist $\xi_{0}, \xi_{1}$ such that $\mathcal{G}_{M_{0}}\left(\xi_{0}\right)>\psi\left(\xi_{0}\right)$ and $\mathcal{G}_{M_{0}}\left(\xi_{1}\right)<\psi\left(\xi_{1}\right)$. Theorem 2.4 then implies that $V(T(s) \psi)<V(\psi)=c$, which contradicts (3.15). So the nonempty $\omega$-limit set should be $\omega\left(w_{0}\right)=\left\{\mathcal{G}_{M_{0}}\right\}$.

4. Diffusion driven interfaces and metastability. The asymptotic behavior of the inviscid Burgers equation is an $\mathrm{N}$-wave [12]. By contrast, solutions of the viscous Burgers equation approach diffusion waves asymptotically in time. That indicates a 
sensitive dependence of the long-time asymptotics for the viscous Burgers equation on the viscosity that we begin analyzing from this section.

Numerical computations for solutions of the viscous Burgers equation were performed using the self-similar version (1.5). This framework has two advantages: first, the solution does not spread in time (thus a smaller computational domain is needed), and second, the time variable $s=\ln (t)$ allows us to compute for long times. The results are presented in Figure 1 for a viscosity $\mu=0.01$ (see section 4.3 for information on the numerical scheme). It is seen that initially $w^{\mu}$ evolves from "oscillatory" initial data $w_{0}$ into a saw-tooth profile. This transition occurs relatively quickly $(s \sim 0.5)$ and is driven by the usual compression-attenuation mechanism of hyperbolic equations. At the next stage the waves interact and eventually produce an approximate $\mathrm{N}$-wave. This takes a longer time $(s \sim 2)$, and this stage is also governed by the convection mechanism mainly (see section 4.3). Once the latter stage is reached, it appears as if there is no dynamical change. A more careful look, though, shows that the negative and positive masses of the solution decrease slowly until eventually the smaller one disappears. This evolution occurs at a far slower time scale. In Figure 1 the negative mass disappears at $s \sim 100$, which, considering that the original time variable is $t=e^{s}$, is an exceptionally long time.

We next analyze the role of diffusion at the intermediate stage driven by wave interactions and then transition from an $\mathrm{N}$-wave to a diffusion wave (for small $s \sim 0.5$ and large $s>2$ for the numerical run of Figure 1). Then, in sections 5 and 6 , we provide a quantitative description of the metastable stage of the evolution.

4.1. Wave interactions. We provide a heuristic analysis valid for solutions emanating from data that at time $s=0$ intersect the axis at finitely many points

$$
\{\xi \in \mathbb{R}: w(\xi, s)=0\}=\left\{g_{1}(s)<g_{2}(s)<\cdots<g_{n}(s)\right\}, \quad s=0,
$$

and the intersections occur transversally

$$
w_{\xi}\left(g_{i}(s), s\right) \neq 0, \quad i=1,2, \ldots, n, \quad s=0 .
$$

For notational convenience, let $g_{0}(s)=-\infty$ and $g_{n+1}(s)=+\infty$. (This is the generic form of solutions that appears in numerical runs after the initial stage $(s \sim 0.5$ in Figure 1); we refer to Figure 2 for such a profile.) Our goal is to track the mechanism of motion of the intersection points.

The points of intersection $g_{i}$ satisfy $w\left(g_{i}(0), 0\right)=0$. Using the implicit function theorem, the curves $g_{i}(s)$ are each defined on a maximal interval $s \in\left[0, S_{i}\right)$ and move with the speed

$$
g_{i}^{\prime}(s)=-\frac{w_{s}\left(g_{i}(s), s\right)}{w_{\xi}\left(g_{i}(s), s\right)} .
$$

On the interval $\left[0, S_{i}\right)$ we have $w_{\xi}\left(g_{i}(s), s\right)>0$, but at the maximal time $S_{i}$ it is $w_{\xi}\left(g_{i}\left(S_{i}\right), S_{i}\right)=0$. Typically, at such times two of the curves will come together and disappear.

Consider the functions

$$
p_{i}(s)=-\int_{-\infty}^{g_{i}(s)} w(\zeta, s) d \zeta=-W\left(g_{i}(s), s\right)
$$

where $-p_{i}(s)$ measures the total mass of $w(\cdot, s)$ to the left of $g_{i}(s)$. Here we define $p_{i}(s)$ with a negative sign since it should represent the invariance variable $p_{0}$ in (1.3) 



FIG. 1. Numerical solution of transformed Burgers (2.1): This numerical solution is generated by the Godunov scheme with discretized viscosity and $\Delta \xi=0.01, \Delta s=0.001, \mu=0.01$.

or $p(s)$ in (2.12). Using (2.13) and the fact that $w\left(g_{i}(s), s\right)=0$, we obtain

$$
-p_{i}^{\prime}(s)=W_{\xi}\left(g_{i}(s), s\right) g_{i}^{\prime}(s)+W_{s}\left(g_{i}(s), s\right)=\mu w_{\xi}\left(g_{i}(s), s\right),
$$

which pinpoints the effect of the diffusion on the mass change across a zero curve $\xi=g_{i}(s)$.

The remainder of our analysis is heuristic in nature. In numerical runs, after an initial transient stage, the usual compression-attenuation mechanism of Burgers 


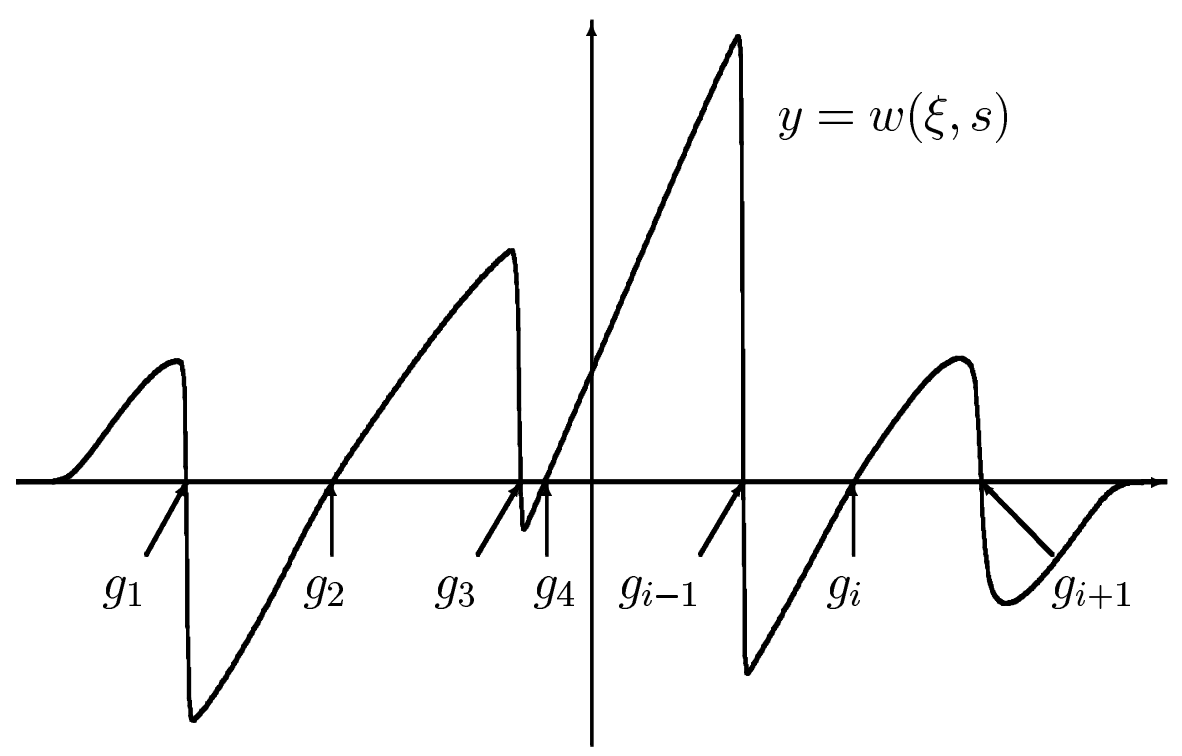

FIG. 2. A profile of the solution at $s=0.5$.

produces solutions that consist entirely of shocks and rarefactions. We will operate under this condition, analyzing solutions whose time sections ( $s$ = constant) consist entirely of shocks and rarefactions and look like that in Figure 2.

The area between two zero curves $g_{i}(s)<g_{i+1}(s)$ (see Figure 2) is given by $A(s)=-\left(p_{i+1}(s)-p_{i}(s)\right)$ and changes in time at a rate

$$
A^{\prime}(s)=-p_{i+1}^{\prime}(s)+p_{i}^{\prime}(s)=\mu w_{\xi}\left(g_{i+1}(s), s\right)-\mu w_{\xi}\left(g_{i}(s), s\right)<0 .
$$

The positive slope at $g_{i}(s)$ represents an approximate rarefaction and may be estimated using the Oleinik estimate, by $0<w_{\xi}\left(g_{i}(s), s\right) \leq 1$. Hence the change of mass across the zero curve $\xi=g_{i}(s)$ satisfies

$$
-p_{i}^{\prime}(s)=\mu w_{\xi}\left(g_{i}(s), s\right) \leq \mu
$$

and is controlled by the diffusion. On the other hand, the negative slope at $g_{i+1}(s)$ will correspond (for $\mu$ small) to a shock profile, and the lower bound for $w_{\xi}$ is of order $O(1 / \mu)$. Hence the mass change across the zero curve $\xi=g_{i+1}(s)$, representing a shock, is of order $O(1)$. This points out the distinct roles of shock and rarefaction profiles: When $w$ is increasing near a zero point, the mass change across the point is controlled by the diffusion and tends to zero as $\mu \rightarrow 0$. By contrast, near a shock profile the mass change is fast and independent of $\mu$. The above estimations remain valid until near the time that two of the curves $g_{i}$ merge and the enclosed area $A(s)$ vanishes.

4.2. Transition from $\mathbf{N}$-wave to diffusion wave. Consider now a solution that emanates from $\mathrm{N}$-wave like data: $w(\xi, 0)<0$ for $\xi<\xi_{0}$ and $w(\xi, 0)>0$ for $\xi>\xi_{0}$ with $w_{\xi}\left(\xi_{0}, 0\right) \neq 0$. We consider the zero-curve $\xi=g(s)$ emanating from the point $g(0)=\xi_{0}$ and satisfying $w(g(s), s)=0$. From the implicit function theorem, the curve $g(s)$ is defined on a maximal interval $[0, S), S>0$. As noted in Remark 2.5 , the number of zeroes is nonincreasing, and for $\mathrm{N}$-wave-like data, as above, it 
cannot happen that $w$ and $w_{\xi}$ vanish at the same point $(\bar{\xi}, \bar{s})$. This implies that either $S=\infty$ or (if $S$ is finite) $g(s) \rightarrow \pm \infty$ as $s \rightarrow S$. In either case the solution retains its $\mathrm{N}$-wave-like form in the interval $[0, S)$.

The infimum $p(s)$ of $(2.12)$ is given by

$$
p(s)=-\int_{-\infty}^{g(s)} w(\zeta, s) d \zeta
$$

From (4.7) we see that

$$
p(s) \geq p(0)-\mu s \quad \text { for } 0<s<S .
$$

We next show that $S \geq \frac{1}{\mu} \min \{p(0), q(0)\}$, which provides an estimate of the transition-time to a diffusion wave. For simplicity, we assume $q(0)>p(0)$ and show that $S \geq p(0) / \mu$. Indeed, if $S<p(0) / \mu$, then from (4.9) we see that $p(s)>0$, which implies that the mass of the negative part of the solution is still present at the time $S$. This contradicts the definition of $S$. The same argument can be made for the positive part of the solution, and we conclude that

$$
\begin{aligned}
& p(s)>p(0)-\mu s, \\
& q(s)>q(0)-\mu s,
\end{aligned} \quad s<\frac{1}{\mu} \min (p(0), q(0)),
$$

where $p(s), q(s)$ are the invariant variables defined in (2.12).

4.3. Comparison of the inviscid and viscous problems. We compare the evolution between the inviscid and the viscous problem for initial data consisting of two separated $\mathrm{N}$-waves

$$
w_{0}(x)=\left\{\begin{array}{cc}
x+10, & -12<x<-8 \\
x, & -\sqrt{2}<x<\sqrt{6} \\
0, & \text { otherwise }
\end{array}\right.
$$

see Figure 3. The method of characteristics gives the exact solution of the inviscid problem: In the original variables $(x, t)$ the solution starts to spread out, and then two inside shocks interact until a single $\mathrm{N}$-wave emerges. In terms of the self-similar variables $(\xi, s)$, one $\mathrm{N}$-wave moves into the origin without changing shape until it collides with the other $\mathrm{N}$-wave, and the interaction results in a new $\mathrm{N}$-wave. The exact solution of the inviscid problem with initial data (4.11) is obtained by tracking the characteristics and is displayed in Figure 3 with solid lines.

The viscous problem (2.1) is solved numerically by the following scheme: Consider a uniform space $\xi_{j+1 / 2}=(j+1 / 2) \Delta \xi$ and time $s_{n}=n \Delta s$ mesh, where $j \in \mathbb{Z}, n \in \mathbb{Z}^{+}$. The approximation of a cell-average $U_{j}^{n}$,

$$
U_{j}^{n} \sim \frac{1}{\Delta \xi} \int_{\xi_{j-1 / 2}}^{\xi_{j+1 / 2}} w\left(\xi, s_{n}\right) d \xi,
$$

is generated by a three-step explicit method,

$$
U_{j}^{n+1}=U_{j}^{n}-\frac{\Delta s}{\Delta \xi}\left(F\left(U_{j}^{n}, U_{j+1}^{n}\right)-F\left(U_{j-1}^{n}, U_{j}^{n}\right)\right)+\mu \frac{\Delta s}{(\Delta \xi)^{2}} D\left(U_{j-1}^{n}, U_{j}^{n}, U_{j+1}^{n}\right),
$$





FIG. 3. Comparison of the inviscid and viscous problems: Solid lines are exact solutions of inviscid problem (2.2), and diamond dots are numerical solutions of viscous problem (2.1) using the Godunov scheme with $\Delta \xi=0.01, \Delta s=0.0005, \mu=0.02$. Solutions are plotted at every other 4 mesh points.

where the numerical flux $F$ is an approximation of

$$
F\left(U_{j}^{n}, U_{j+1}^{n}\right) \sim \frac{1}{\Delta s} \int_{s_{n}}^{s_{n+1}} \frac{1}{2}\left(w^{2}-\xi_{j+1 / 2} w\right) d s
$$


and the diffusion term is discretized by

$$
D\left(U_{j-1}^{n}, U_{j}^{n}, U_{j+1}^{n}\right)=U_{j-1}^{n}-2 U_{j}^{n}+U_{j+1}^{n} .
$$

Since the flux of the self-similar Burgers (2.1) depends on the space variable, the solution of the Riemann problem increases exponentially along the characteristics $w(\xi(s), s)=w(\xi(0), 0) e^{s / 2}$. The characteristics are not straight lines, and schemes using Riemann solver, like the Godunov scheme (see [10]), should take that into account. Here we consider a numerical flux

$$
F\left(U_{j}^{n}, U_{j+1}^{n}\right)=\left\{\begin{aligned}
I\left(U_{j+1}^{n}, \bar{\xi}\right) & \text { if } \lambda\left(U_{j}^{n}, \bar{\xi}\right)+\lambda\left(U_{j+1}^{n}, \bar{\xi}\right) \leq 0, \lambda\left(U_{j+1}^{n}, \bar{\xi}\right) \leq 0, \\
I\left(U_{j}^{n}, \bar{\xi}\right) & \text { if } \lambda\left(U_{j}^{n}, \bar{\xi}\right)+\lambda\left(U_{j+1}^{n}, \bar{\xi}\right)>0, \lambda\left(U_{j}^{n}, \bar{\xi}\right)>0, \\
-3 \bar{\xi}^{2} / 8 & \text { if } \lambda\left(U_{j}^{n}, \bar{\xi}\right)<0, \lambda\left(U_{j+1}^{n}, \bar{\xi}\right)>0
\end{aligned}\right.
$$

where $\bar{\xi}=\xi_{j+1 / 2}$ and $\lambda$ is the wave speed

$$
\lambda(U, \xi)=U-\xi / 2
$$

$I(U, \xi)$ is an approximation of the line integral of (4.13) for a shock wave which is given by

$$
I(U, \xi)=\frac{1}{2} U^{2}\left(e^{\Delta s}-1\right)-\xi U\left(e^{\Delta s / 2}-1\right),
$$

and we can easily check that the rarefaction wave centered at $\bar{\xi}$ has constant value $w=-3 \bar{\xi}^{2} / 8$ along the vertical line $\xi=\bar{\xi}$.

In the computation of Figure 3 the mesh size is $\Delta \xi=0.01$, the time step is $\Delta s=0.0005$, and the viscosity $\mu=0.02$. The numerical solution is displayed with diamond dots. The first column indicates that the solution of the viscous problem with small viscosity is close to the solution of the inviscid problem, until the solution reaches the state of single N-wave (which is a steady state for the inviscid problem (1.6)). This stage of the evolution is dominated by convection. Subsequently, the diffusion becomes dominant, and the solution evolves slowly until it reaches the asymptotic state of a diffusion wave (a steady state for (1.5)).

Remark 4.1 (monitoring the viscosity of a numerical scheme). Numerical schemes for inviscid problems introduce numerical viscosity. A classical example is the first order Lax-Friedrich scheme for the linear equation $u_{t}+A u_{x}=0$, which is actually a second order scheme for $u_{t}+A u_{x}=\varepsilon u_{x x}$ with a numerical viscosity

$$
\varepsilon=\left(1-\frac{(\Delta t)^{2}}{(\Delta x)^{2}} A^{2}\right) \frac{(\Delta x)^{2}}{2 \Delta t} .
$$

For nonlinear equations it is hard to have such an explicit control of the numerical viscosity. For that reason we opted to use a scheme based on the parabolic equation, using Godunov for the convection term and a discretization for the diffusion term.

Nevertheless, it is possible that the numerical viscosity $\varepsilon$ can be different from the purported one $\mu$. Under the assumption that numerical viscosity is the only factor that causes the area change of numerically approximate $\mathrm{N}$-waves (an assumption clearly valid at the level of the differential equation), we can measure the numerical viscosity, using the formula (4.7), by measuring the area change and the slope at the zero point. Consider, for example, the initial data

$$
w_{0}(\xi)=\left\{\begin{array}{rr}
x, & -2<x<2 \\
0, & \text { otherwise }
\end{array}\right.
$$


TABLE 1

Numerical viscosity of (4.12): Initial data (4.19), $\mu=0.05, \Delta \xi=0.01, \Delta s=0.000667$.

\begin{tabular}{ccccc}
\hline$s^{n}-1<s<s^{n}$ & \multicolumn{1}{c}{$\max \left(U^{n}\right)$} & $P\left(s^{n}\right)=\sum_{j<0} U_{j}^{n}$ & $P\left(s^{n}\right)-P\left(s^{n}-1\right)$ & $\Delta P / \bar{w}_{\xi} \sim \varepsilon$ \\
\hline $5<s<6$ & 1.578890 & -1.686368 & 0.050618 & 0.050003 \\
$10<s<11$ & 1.425409 & -1.433271 & 0.050620 & 0.050003 \\
$20<s<21$ & 1.068703 & -0.927091 & 0.050614 & 0.050003 \\
$35<s<36$ & 0.303368 & -0.186434 & 0.043840 & 0.050083 \\
\hline $37<s<38$ & 0.189594 & -0.110107 & 0.035890 & 0.050087 \\
$39<s<40$ & 0.100160 & -0.055559 & 0.024223 & 0.049915 \\
$41<s<42$ & 0.045148 & -0.024340 & 0.012980 & 0.049550 \\
$43<s<44$ & 0.018336 & -0.009749 & 0.005776 & 0.049215 \\
\hline $45<s<46$ & 0.007074 & -0.003739 & 0.002319 & 0.049029 \\
$55<s<56$ & 0.000052 & -0.000027 & 0.000017 & 0.048896 \\
$70<s<71$ & $3.135604 \mathrm{e}-08$ & $-1.651307 \mathrm{e}-08$ & $1.054758 \mathrm{e}-08$ & 0.048895 \\
$90<s<91$ & $1.812541 \mathrm{e}-12$ & $-9.858833 \mathrm{e}-13$ & $5.406505 \mathrm{e}-13$ & 0.048895 \\
\hline
\end{tabular}

As the area of the negative part is $\int_{-\infty}^{0} w(\zeta, s) d \zeta$, we consider $P\left(s^{n}\right)=\sum_{j<0} \Delta \xi U_{j}^{n}$. From (4.7) it is natural to define the numerical viscosity as

$$
\varepsilon\left(s^{n}\right)=\frac{P\left(s^{n}\right)-P\left(s^{n-1}\right)}{\Delta s \bar{w}_{\xi}},
$$

where the slope at the zero point is approximated by $\bar{w}_{\xi}=\frac{U_{1}^{n}-U_{-1}^{n}}{2 \Delta \xi}$. In Table 1 we present the measured numerical viscosity for the scheme (4.12) with initial data (4.19) and viscosity $\mu=0.05$. It is seen that the numerical viscosity represents the purported viscosity very well, $\varepsilon \sim \mu$, but as the solution decreases (in the second part of the table), the viscosity also decreases slightly. In the last part the solution is almost zero, and the numerical viscosity remains constant at the value around $\varepsilon \sim 0.049$.

5. Diffusive $\mathbf{N}$-waves. The Cole-Hopf transformation implies that $u(x, t)$ is a solution of (1.1) if and only if

$$
\varphi(x, t)=e^{-\frac{1}{2 \mu} \int_{-\infty}^{x} u(x, t) d x}
$$

solves the heat equation

$$
\varphi_{t}=\mu \varphi_{x x}
$$

Note that (5.1) implies

$$
\varphi(-\infty, t)=1, \quad u=-2 \mu \frac{\varphi_{x}}{\varphi}, \quad \int_{a}^{b} u(x, t) d x=-2 \mu \ln (\varphi(b, t) / \varphi(a, t))
$$

and allows us to compute the mass of $u$. Whitham [21] uses the transformation to produce a special solution of a diffusive $\mathrm{N}$-wave with equal positive and negative mass. In this section we give an extension to this construction and compute a special solution of a diffusive $\mathrm{N}$-wave with possibly unequal positive and negative masses. This solution characterizes the transition from a diffusive $\mathrm{N}$-wave to a diffusion wave, observed during the late-time response of Burgers.

We consider the special potential

$$
\varphi_{p, q}(x, t)=1+A \sqrt{\frac{t_{0}}{t}} e^{-x^{2} / 4 \mu t}-B \frac{1}{\sqrt{\pi}} \int_{-\infty}^{\frac{x}{\sqrt{4 \mu t}}} e^{-\zeta^{2}} d \zeta
$$


and the corresponding solution of Burgers

$$
u_{p, q}(x, t)=-2 \mu \frac{\left(\varphi_{p, q}\right)_{x}}{\varphi_{p, q}}=\frac{\frac{x}{t} A \sqrt{\frac{t_{0}}{t}} e^{-x^{2} / 4 \mu t}+\sqrt{\frac{\mu}{t}} \frac{B}{\sqrt{\pi}} e^{-x^{2} / 4 \mu t}}{1+A \sqrt{\frac{t_{0}}{t}} e^{-x^{2} / 4 \mu t}-B \frac{1}{\sqrt{\pi}} \int_{-\infty}^{\frac{x}{\sqrt{4 \mu t}}} e^{-\zeta^{2} d \zeta}} .
$$

Recall that the positive and negative mass of the solution $u$ are computed by

$$
p(t)=-\inf _{x} \int_{-\infty}^{x} u_{p, q}(y, t) d y, \quad q(t)=\sup _{x} \int_{x}^{\infty} u_{p, q}(y, t) d y .
$$

$p(t), q(t)$ are not invariant for the viscous Burgers, but the total mass $(q(t)-p(t))$ is. The constants $A>0$ and $B$ in (5.4) will be determined so that the positive and negative masses at a given time $t_{0}$ are prescribed positive constants $p$ and $q$, that is, $p\left(t_{0}\right)=p$ and $q\left(t_{0}\right)=q$.

This takes a lengthy computation that we outline below. To fit the total mass $M=\int u_{p, q}(y, t) d y=q-p$, we use (5.3) and obtain

$$
\int_{-\infty}^{\infty} u_{p, q}(y, t) d y=-2 \mu \ln (1-B)=q-p .
$$

Hence $B=1-e^{(p-q) / 2 \mu}$. Note that $B>0$ for $p<q$ and $B<0$ for $q<p$; in either case $1-B>0$.

Clearly, $u<0$ for $x<x_{0}(t)=-t \sqrt{\mu} B / \sqrt{t_{0} \pi} A$, and $u>0$ for $x>x_{0}(t)$. The negative mass is computed from (5.3):

$$
\begin{aligned}
p(t) & =-\inf _{x} \int_{-\infty}^{x} u_{p, q}(y, t) d y=2 \mu \ln \frac{\varphi\left(x_{0}(t), t\right)}{\varphi(-\infty, t)} \\
& =2 \mu \ln \left(1+A \sqrt{\frac{t_{0}}{t}} e^{-\frac{t}{t_{0}}\left(\frac{B}{\sqrt{4 \pi} A}\right)^{2}}-\frac{B}{\sqrt{\pi}} \int_{-\infty}^{-\sqrt{\frac{t}{t_{0}}} \frac{B}{\sqrt{4 \pi} A}} e^{-\zeta^{2}} d \zeta\right) .
\end{aligned}
$$

The requirement $p\left(t_{0}\right)=p$ gives the equation

$$
A e^{-\left(\frac{B}{\sqrt{4 \pi} A}\right)^{2}}=e^{p / 2 \mu}-1+\frac{B}{\sqrt{\pi}} \int_{-\infty}^{-\frac{B}{\sqrt{4 \pi} A}} e^{-\zeta^{2}} d \zeta
$$

We give an approximate solution of (5.8). Note that $A>e^{p / 2 \mu}-1+c B$ for some $0<c<1$, which in turn gives the estimates

$$
A=O\left(e^{\frac{p}{2 \mu}}\right), \quad \frac{B}{A}=O\left(e^{-\frac{p}{2 \mu}}+e^{-\frac{q}{2 \mu}}\right), \quad \text { as } \mu \rightarrow 0 .
$$

Now we can rewrite (5.8) and use Taylor expansion to obtain

$$
\begin{aligned}
e^{p / 2 \mu}-1 & =\left.A\left(e^{-\rho^{2}}-2 \rho \int_{-\infty}^{-\rho} e^{-\zeta^{2}} d \zeta\right)\right|_{\rho=\frac{B}{\sqrt{4 \pi} A}} \\
& =\left.A\left(1-\sqrt{\pi} \rho+O\left(\rho^{2}\right)\right)\right|_{\rho=\frac{B}{\sqrt{4 \pi} A}}=A\left(1-\frac{1}{2} \frac{B}{A}+O\left(\left(\frac{B}{A}\right)^{2}\right)\right) .
\end{aligned}
$$

We conclude that

$$
B=1-e^{(p-q) / 2 \mu}, \quad A=e^{p / 2 \mu}-1+B\left[\frac{1}{2}+O\left(e^{-\frac{p}{2 \mu}}+e^{-\frac{q}{2 \mu}}\right)\right]
$$


and that $A=e^{p / 2 \mu}+O(B)$ as $\mu \rightarrow 0$.

Next we consider the behavior of $u_{p, q}$ as the viscosity $\mu \rightarrow 0$. We write this $u_{p, q}$ in the form

$$
u_{p, q}(x, t)=\frac{\frac{x}{t}+\frac{B}{A} \sqrt{\frac{\mu}{\pi t_{0}}}}{1+\sqrt{\frac{t}{t_{0}}} \frac{e^{\frac{x^{2}}{4 \mu t}}}{A}\left(1-B \frac{1}{\sqrt{\pi}} \int_{-\infty}^{x / \sqrt{4 \mu t}} e^{-y^{2}} d y\right)} .
$$

It is clear that

$$
\begin{aligned}
\frac{x}{t}+\frac{B}{A} \sqrt{\frac{\mu}{\pi t_{0}}} & \rightarrow 0, \\
\frac{1}{A} e^{\frac{x^{2}}{4 \mu t}}\left(1-B \frac{1}{\sqrt{\pi}} \int_{-\infty}^{x / \sqrt{4 \mu t}} e^{-y^{2}} d y\right) & \sim\left\{\begin{array}{cc}
\frac{1}{A} e^{\frac{x^{2}}{4 \mu t}}, & x<0, \\
\frac{1-B}{A} e^{\frac{x^{2}}{4 \mu t}}, & x>0,
\end{array}\right. \\
& \sim \begin{cases}e^{\frac{1}{4 \mu t}\left(x^{2}-2 p t\right)}, & x<0, \\
e^{\frac{1}{4 \mu t}\left(x^{2}-2 q t\right)}, & x>0,\end{cases}
\end{aligned}
$$

and, as a result,

$$
u_{p, q}(x, t) \sim\left\{\begin{aligned}
0, & x<-\sqrt{2 p t} \\
x / t, & -\sqrt{2 p t}<x<\sqrt{2 q t}, \\
0, & x>\sqrt{2 q t}
\end{aligned}\right.
$$

as $\mu \rightarrow 0$. This validates the terminology diffusive $\mathrm{N}$-wave for $u_{p, q}$.

The long-time behavior of $u_{p, q}(x, t)$ is also easily computed. A simple inspection shows that as $t \rightarrow \infty$

$$
\varphi_{p, q}(x, t) \sim 1-\frac{B}{\sqrt{4 \pi \mu t}} \int_{-\infty}^{x} e^{-y^{2} / 4 \mu t} d y,
$$

within leading order, and that $u_{p, q}$ has the structure of a diffusion wave of mass $M$.

If $M>0$, the area of the negative part of the solution diminishes as $t \rightarrow \infty$. The time at which most of the negative area has almost disappeared can be estimated as follows: First, (5.7) is written in the form

$$
e^{p(t) / 2 \mu}=1+A \sqrt{\frac{t_{0}}{t}} e^{-\frac{t}{t_{0}} \frac{B^{2}}{4 \pi A^{2}}}-\frac{B}{\sqrt{\pi}} \int_{-\infty}^{-\sqrt{\frac{t}{t_{0}}} \frac{B}{\sqrt{4 \pi} A}} e^{-\zeta^{2}} d \zeta .
$$

We substitute $t=t_{0} A^{2} / B^{2}$ and obtain

$$
e^{p(t) / 2 \mu}=1+|B| e^{-\frac{1}{4 \pi}}-\frac{B}{\sqrt{\pi}} \int_{-\infty}^{-\frac{\operatorname{sign}(\mathrm{B})}{\sqrt{4 \pi}}} e^{-\zeta^{2}} d \zeta .
$$

If $q>p$, then $B \sim 1$, and we get

$$
e^{p(t) / 2 \mu} \sim 1+e^{-\frac{1}{4 \pi}}-\frac{1}{\sqrt{\pi}} \int_{-\infty}^{-\frac{1}{\sqrt{4 \pi}}} e^{-\zeta^{2}} d \zeta \sim 1.579
$$


so that

$$
p\left(t_{0} A^{2} / B^{2}\right) \sim 2 \mu \ln (1.579) \sim 0.913 \mu .
$$

On the other hand, if $M<0$ (equivalently, $p>q$ ), it is the area of the positive part of the solution that diminishes. The critical time is now estimated as follows: For $p>q$ and $\mu \ll 1$, we have $B \sim-e^{(p-q) / 2 \mu}<0$. Dividing both sides of (5.12) by $|B|$, we obtain

$$
e^{p(t) / 2 \mu} /|B|=1 /|B|+e^{-\frac{1}{4 \pi}}+\frac{1}{\sqrt{\pi}} \int_{-\infty}^{\frac{1}{\sqrt{4 \pi}}} e^{-\zeta^{2}} d \zeta
$$

In turn,

$$
e^{q(t) / 2 \mu}=e^{(p(t)-(p-q)) / 2 \mu} \sim e^{-\frac{1}{4 \pi}}+\frac{1}{\sqrt{\pi}} \int_{-\infty}^{\frac{1}{\sqrt{4 \pi}}} e^{-\zeta^{2}} d \zeta \sim 1.579
$$

and

$$
q\left(t_{0} A^{2} / B^{2}\right) \sim 0.913 \mu .
$$

Remark 5.1. Because of the invariance of Burgers under translations $t \rightarrow t+a$, the functions $\varphi_{p, q}(x, t+1)$ and $u_{p, q}(x, t+1)$ are also special solutions associated to diffusive $\mathrm{N}$-waves. The latter can be expressed (for $t_{0}=1$ ) in the form

$$
\begin{aligned}
& \varphi_{p, q}(x, t+1)=\psi_{p, q}\left(\frac{x}{\sqrt{4 \mu(t+1)}}, t\right) \\
& u_{p, q}(x, t+1)=\sqrt{\frac{\mu}{t+1}} v_{p, q}\left(\frac{x}{\sqrt{4 \mu(t+1)}}, t\right)
\end{aligned}
$$

where

$$
\begin{aligned}
& \psi_{p, q}(\xi, t)=1-\frac{B}{\sqrt{\pi}} \int_{-\infty}^{\xi} e^{-\zeta^{2}} d \zeta+A \frac{1}{\sqrt{t+1}} e^{-\xi^{2}}, \\
& v_{p, q}(\xi, t)=\frac{\frac{B}{\sqrt{\pi}} e^{-\xi^{2}}+2 A \frac{1}{\sqrt{t+1}} \xi e^{-\xi^{2}}}{1-\frac{B}{\sqrt{\pi}} \int_{-\infty}^{\xi} e^{-\zeta^{2}} d \zeta+A \frac{1}{\sqrt{t+1}} e^{-\xi^{2}}} .
\end{aligned}
$$

The form (5.13) of the diffusive $\mathrm{N}$-waves motivates an explicit solution for the Cauchy problem of the viscous Burgers equation, which is carried out in section 6. A survey of this solution and comparison with $v_{p, q}$ indicates that while the diffusion wave is the $t \rightarrow \infty$ asymptotic profile for the viscous Burgers, the diffusive $\mathrm{N}$-wave $u_{p, q}$ gives a more accurate description of the behavior in the large time regime, which encompasses the very long-time behavior and the leading order correction and is valid for a substantially longer time interval.

6. An explicit solution of the viscous Burgers equation. The objective of this section is to derive an explicit solution of the Cauchy problem for the viscous Burgers equation

$$
\begin{array}{ll}
u_{t}+u u_{x}=\mu u_{x x}, & x \in \mathbb{R}, \quad \mu, t>0, \\
u(x, 0)=u_{0}(x), & x \in \mathbb{R} .
\end{array}
$$


Our approach hinges on the invariance properties of the viscous Burgers equation, a self-similar variant of the Cole-Hopf transformation, and the exact solvability of a Fokker-Planck-type of equation. It yields an explicit solution of (6.1) in terms of Hermite polynomials.

Step 1. First, we apply to (6.1) the change of variables

$$
u(x, t)=\sqrt{\frac{\mu}{t+1}} v\left(\frac{x}{\sqrt{4 \mu(t+1)}}, t\right) .
$$

Then the function $v(\xi, t)$ of the similarity variable $\xi=\frac{x}{\sqrt{4 \mu(t+1)}}$ satisfies the Cauchy problem

$$
\begin{array}{ll}
(t+1) v_{t}+\left(-\frac{1}{2} \xi v+\frac{1}{4} v^{2}\right)_{\xi}=\frac{1}{4} v_{\xi \xi}, & \xi \in \mathbb{R}, \quad t>0, \\
v(\xi, 0)=v_{0}(\xi), & \xi \in \mathbb{R},
\end{array}
$$

with initial data

$$
v_{0}(\xi)=\frac{1}{\sqrt{\mu}} u_{0}(\sqrt{4 \mu} \xi) .
$$

The transformation is motivated by the invariance properties of the viscous Burgers equation and the form of the special solutions termed diffusive $\mathrm{N}$-waves in section 5 . Despite their similarity, the transformation (1.4) used in sections 2-4 differs in the dependence on viscosity and should not be confused with (6.2). In problem (6.3) the sole dependence on viscosity is through the initial data.

Step 2. We apply to (6.3) a variant of the Cole-Hopf transformation. Let

$$
V(\xi, t)=\int_{-\infty}^{\xi} v(\zeta, t) d \zeta
$$

and introduce $\psi(\xi, t)$ so that

$$
V=-\ln \psi, \quad v=-\frac{\psi_{\xi}}{\psi} .
$$

A calculation shows that $V$ satisfies

$$
(t+1) V_{t}-\frac{1}{2} \xi V_{\xi}+\frac{1}{4} V_{\xi}^{2}=\frac{1}{4} V_{\xi \xi},
$$

and $\psi$ satisfies the initial value problem

$$
4(t+1) \psi_{t}=\psi_{\xi \xi}+2 \xi \psi_{\xi}, \quad \xi \in \mathbb{R}, t>0,
$$

with data

$$
\begin{aligned}
\psi(\xi, 0) & =\bar{\psi}(\xi):=e^{-V_{0}(\xi)} \\
\text { where } \quad V_{0}(\xi) & =\int_{-\infty}^{\xi} v_{0}(\zeta) d \zeta=\frac{1}{2 \mu} \int_{-\infty}^{\sqrt{4 \mu} \xi} u_{0}(y) d y \\
& =\frac{M}{2 \mu}-\frac{1}{2 \mu} \int_{\sqrt{4 \mu} \xi}^{\infty} u_{0}(y) d y
\end{aligned}
$$


Step 3. Next we solve the initial value problem consisting of (6.7) with initial data

$$
\psi(\xi, 0)=\psi_{0}(\xi), \quad \text { with } \quad e^{\frac{\xi^{2}}{2}} \psi_{0} \in L^{2}(\mathbb{R})
$$

via separation of variables. This leads to the issue of finding the eigenvalues and eigenfunctions of the boundary value problem

$$
g^{\prime \prime}+2 \xi g^{\prime}=\lambda g, \quad-\infty<\xi<\infty .
$$

The problem (6.10) turns out to have a discrete spectrum, associated with the Hermite polynomials.

The Hermite polynomials (see Szegö [19, Chapter V]) are the solutions $y=H_{n}(\xi)$, $n=0,1,2, \ldots$, of the boundary value problem

$$
y^{\prime \prime}-2 \xi y^{\prime}+2 n y=0, \quad-\infty<\xi<\infty .
$$

$H_{n}$ are polynomials of degree $n$ and are generated from the relation

$$
H_{n}(\xi)=(-1)^{n} e^{\xi^{2}} \frac{d^{n}}{d \xi^{n}}\left(e^{-\xi^{2}}\right)
$$

The first few of them are $H_{0}=1, H_{1}=2 \xi, H_{2}=4 \xi^{2}-2$, and so on. They satisfy the orthogonality conditions

$$
\int_{-\infty}^{\infty} H_{m}(\xi) H_{n}(\xi) e^{-\xi^{2}} d \xi=2^{n} n ! \sqrt{\pi} \delta_{n m}
$$

and the system $\left\{H_{n}(\xi) e^{-\frac{\xi^{2}}{2}}\right\}_{n=0}^{\infty}$ is a complete orthogonal system in $L^{2}(\mathbb{R})$.

Using these properties, it can be seen that the eigenvalues and eigenfunctions of (6.10) are $\lambda_{n}=-2(n+1)$ and $g_{n}(\xi)=e^{-\xi^{2}} H_{n}(\xi), n=0,1,2, \ldots$ Moreover, the solution of (6.7)-(6.9) is given in the form of a series

$$
\psi(\xi, t)=\sum_{n=0}^{\infty} a_{n}(t+1)^{-\frac{n+1}{2}} H_{n}(\xi) e^{-\xi^{2}},
$$

where $a_{n}$ are determined by

$$
\psi_{0}(\xi) e^{\frac{\xi^{2}}{2}}=\sum_{n=0}^{\infty} a_{n} H_{n}(\xi) e^{-\frac{\xi^{2}}{2}} .
$$

For $\psi_{0} e^{\frac{\xi^{2}}{2}} \in L^{2}(\mathbb{R})$ this problem is solvable, and the Fourier-Hermite coefficients are determined by the formula

$$
a_{n}=\frac{1}{2^{n} n ! \sqrt{\pi}} \int_{-\infty}^{\infty} \psi_{0}(\xi) H_{n}(\xi) d \xi .
$$

Remark 6.1. The Hermite polynomials also appear in the eigenfunctions of the eigenvalue problem (sometimes called Hermite functions)

$$
z^{\prime \prime}+\left(2 n+1-\xi^{2}\right) z=0, \quad z_{n}(\xi)=H_{n}(\xi) e^{-\frac{\xi^{2}}{2}}, \quad n=0,1,2, \ldots
$$


which is associated with the problem of the harmonic oscillator in quantum mechanics. The eigenfunctions of the problem at hand are different from the ones above. The operator in (6.10) can be thought of as the integrated version of a Fokker-Planck-type operator.

Now consider the problem (6.7) with initial data

$$
\psi(\xi, 0)=\bar{\psi}(\xi), \quad \bar{\psi}(\xi) \rightarrow a \text { as } \xi \rightarrow-\infty, \quad \bar{\psi}(\xi) \rightarrow b \text { as } \xi \rightarrow \infty .
$$

The steady states $\psi^{\infty}(\xi)$ of $(6.7)-(6.13)$ solve

$$
\psi_{\xi \xi}^{\infty}+2 \xi \psi_{\xi}^{\infty}=0, \quad \psi^{\infty}(-\infty)=a, \quad \psi^{\infty}(\infty)=b,
$$

and are given by

$$
\psi^{\infty}(\xi)=a+\frac{b-a}{\sqrt{\pi}} \int_{-\infty}^{\xi} e^{-\zeta^{2}} d \zeta
$$

By superposition, it is possible to solve (6.7) with initial data

$$
\bar{\psi}(\xi)=\psi^{\infty}(\xi)+\psi_{0}(\xi), \quad \text { where } \quad \psi_{0} e^{\frac{\xi^{2}}{2}} \in L^{2}(\mathbb{R}) .
$$

Its solution is given in the form

$$
\psi(\xi, t)=\psi^{\infty}(\xi)+\sum_{n=0}^{\infty} a_{n}(t+1)^{-\frac{n+1}{2}} H_{n}(\xi) e^{-\xi^{2}}
$$

where the Fourier coefficients $a_{n}$ are computed from (6.12).

Step 4. Returning now to Burgers, we assume that $u_{0}(x)=O\left(e^{-x^{2}}\right)$ as $|x| \rightarrow \infty$. Then $v_{0}(\xi)=O\left(e^{-\xi^{2}}\right)$ as $|\xi| \rightarrow \infty$. (The orders will, in general, depend on the viscosity.) The initial data $\bar{\psi}$ are given in (6.8) and satisfy $\bar{\psi}(-\infty)=1$ and $\bar{\psi}(\infty)=$ $e^{-\frac{M}{2 \mu}}$. We define the associated diffusion wave

$$
\begin{aligned}
\psi_{M}^{\infty} & =1-\frac{1-e^{-\frac{M}{2 \mu}}}{\sqrt{\pi}} \int_{-\infty}^{\xi} e^{-\zeta^{2}} d \zeta \\
& =e^{-\frac{M}{2 \mu}}+\frac{1-e^{-\frac{M}{2 \mu}}}{\sqrt{\pi}} \int_{\xi}^{\infty} e^{-\zeta^{2}} d \zeta .
\end{aligned}
$$

Consider

$$
\psi_{0}=\bar{\psi}-\psi_{M}^{\infty}=e^{-V_{0}}-\psi_{M}^{\infty},
$$

and note that $\psi_{0}$ can be expressed as

$$
\begin{aligned}
\psi_{0}(\xi) & =e^{-\int_{-\infty}^{\xi} v_{0} d \zeta}-1+\frac{1-e^{-\frac{M}{2 \mu}}}{\sqrt{\pi}} \int_{-\infty}^{\xi} e^{-\zeta^{2}} d \zeta \\
& =e^{-\frac{M}{2 \mu}+\int_{\xi}^{\infty} v_{0} d \zeta}-e^{-\frac{M}{2 \mu}}-\frac{1-e^{-\frac{M}{2 \mu}}}{\sqrt{\pi}} \int_{\xi}^{\infty} e^{-\zeta^{2}} d \zeta .
\end{aligned}
$$

Using the inequalities $\left|e^{x}-1\right| \leq 2 x$ for $|x| \ll 1$ and the decay $v_{0}(\xi)=O\left(e^{-\xi^{2}}\right)$ as $|\xi| \rightarrow \infty$, we see that $\psi_{0}(\xi)=O\left(e^{-\xi^{2}}\right)$ as $|\xi| \rightarrow \infty$. We apply the results of the 
previous section and see that $\psi$ is given by (6.15). As a result, the solution of (6.1) is given by the formula

$$
\begin{aligned}
& u(x, t)=\sqrt{\frac{\mu}{t+1}} v\left(\frac{x}{\sqrt{4 \mu(t+1)}}, t\right) \\
& \text { where } v(\xi, t)=-\frac{\partial_{\xi} \psi_{M}^{\infty}+\sum_{n=0}^{\infty} a_{n}(t+1)^{-\frac{n+1}{2}} \partial_{\xi}\left(H_{n}(\xi) e^{-\xi^{2}}\right)}{\psi_{M}^{\infty}+\sum_{n=0}^{\infty} a_{n}(t+1)^{-\frac{n+1}{2}} H_{n}(\xi) e^{-\xi^{2}}} .
\end{aligned}
$$

$\psi_{M}^{\infty}$ is given in (6.16), and the coefficients $a_{n}$ are computed by

$$
a_{n}=\frac{1}{2^{n} n ! \sqrt{\pi}} \int_{-\infty}^{\infty}\left(e^{-V_{0}}-\psi_{M}^{\infty}\right) H_{n} d \xi .
$$

In view of the form of the Hermite polynomials, the coefficients $a_{n}$ may also be expressed in terms of moments of the function $\psi_{0}=e^{-V_{0}}-\psi_{M}^{\infty}$.

An inspection of (6.17) shows that as $t \rightarrow \infty$

$$
v \sim-\frac{\partial_{\xi} \psi_{M}^{\infty}}{\psi_{M}^{\infty}}
$$

which is the asymptotic profile of a diffusion wave of mass $M$. The next order approximation is (recall that $H_{0}=1$ )

$$
v(\xi, t) \sim-\frac{\partial_{\xi} \psi_{M}^{\infty}-2 a_{0}(t+1)^{-\frac{1}{2}} \xi e^{-\xi^{2}}}{\psi_{M}^{\infty}+a_{0}(t+1)^{-\frac{1}{2}} e^{-\xi^{2}}},
$$

which is that of a diffusive $\mathrm{N}$-wave (compare with (5.14)). The coefficient $a_{0}$ is computed by the formula

$$
a_{0}=\frac{1}{\sqrt{\pi}} \int_{-\infty}^{\infty} e^{-V_{0}}-\psi_{M}^{\infty} d \xi
$$

\section{REFERENCES}

[1] S. Angenent, The zero set of a solution of a parabolic equation, J. Reine Angew. Math., 390 (1988), pp. 79-96.

[2] C. M. Dafermos, Generalized characteristics and the structure of solutions of hyperbolic conservation laws, Indiana Univ. Math. J., 26 (1977), pp. 1097-1119.

[3] C. M. Dafermos, Asymptotic behavior of solutions of evolution equations, in Nonlinear Evolution Equations, M. G. Crandall, ed., Academic Press, New York, 1978, pp. 103-124.

[4] C. M. Dafermos, Hyperbolic Conservation Laws in Continuum Physics, Springer-Verlag, New York, 2000.

[5] M. Escobedo And E. Zuazua, Large time behavior for convection-diffusion equations in $R^{N}$, J. Funct. Anal., 100 (1991), pp. 119-161.

[6] M. Escobedo, J. L. Vazquez, And E. Zuazua, Asymptotic behaviour and source-type solutions for a diffusion-convection equation, Arch. Ration. Mech. Anal., 124 (1993), pp. 43-65.

[7] E. Hopf, The partial differential equation $u_{t}+u u_{x}=\mu u_{x x}$, Comm. Pure Appl. Math., 3 (1950), pp. 201-230.

[8] S. N. KRUzhKov, First order quasilinear equations in several independent variables, Math. USSR Sb. 10 (1970), pp. 217-243. 
[9] S. N. KruzhKov, Quasilineare Gleichungen erster Ordnung mit mehreren unabhaengigen Veraenderlichen, Mat. Sb., 123 (1970), pp. 228-255.

[10] R. J. LeVeque, Numerical Methods for Conservation Laws, Lectures in Mathematics ETH Zürich, Birkhäuser-Verlag, Basel, 1990.

[11] G. Lieberman, Second Order Parabolic Differential Equations, World Scientific, River Edge, NJ, 1996.

[12] T.-P. LiU, Invariants and asymptotic behavior of solutions of a conservation law, Proc. Amer. Math. Soc., 71 (1978), pp. 227-231.

[13] T.-P. LiU And M. Pierre, Source-solutions and asymptotic behavior in conservation laws, J. Differential Equations, 51 (1984), pp. 419-441.

[14] T.-P. Liu, A. Matsumura, And K. Nishinara, Behaviors of solutions for the Burgers equation with boundary corresponding to rarefaction waves, SIAM J. Math. Anal., 29 (1998), pp. 293-308.

[15] H. Matano, Nonincrease of the lap number of a solution for a one-dimensional semi-linear parabolic equation, J. Fac. Sci. Univ. Tokyo Sect. IA Math., 29 (1982), pp. 401-441.

[16] O. Oleinik, Discontinuous solutions of nonlinear differential equations, Uspekhi Mat. Nauk (N.S.), 12 (1957), pp. 3-73 (in Russian).

[17] O. Oleinik, Discontinuous solutions of nonlinear differential equations, Amer. Math. Soc. Transl. (2), 26 (1963), pp. 95-172.

[18] D. H. SATtinger, On the total variation of solutions of parabolic equations, Math. Ann., 183 (1969), pp. 78-92.

[19] G. Szegö, Orthogonal Polynomials, Amer. Math. Soc. Colloq. Publ. 23, AMS, Providence, RI, 1939.

[20] L. TARTAR, Une introduction a la théorie matheématique des systèmes hyperboliques de lois de conservation, Pubblicazioni CNR Instituto di Analisi Numerica, 682, Pavia, Italy, 1989.

[21] G. Whitham, Linear and Nonlinear Waves, Pure Appl. Math., Wiley Interscience, New York, 1974. 